\title{
“LA VARIABILIDAD PLUVIOMÉTRICA EN LA PROVINCIA DEL CHACO DURANTE EL PERÍODO 1955 - 2009"
}

Prof. Gómez, Claudia Verónica (Dpto. de Geografía-Facultad de Humanidades-UNNE) Prof. Pérez, María Emilia (Dpto. de Geografía-Facultad de Humanidades-UNNE)

\section{Resumen:}

La precipitación conjuntamente con la temperatura son los elementos del clima que mayor interés despierta en la comunidad científica puesto que influyen definitivamente en las actividades económicas de la población.

La Precipitación, sin embargo, se caracteriza por ser enormemente variable, característica que dificulta establecer un patrón en su comportamiento temporal o espacial que contribuya en la toma de decisiones económicas y sociales adecuadas.

En estudios anteriores que tratan sobre la dinámica pluviométrica en la provincia del Chaco se han detectado un gradiente pluviométrico que aumenta de Oeste-Este, una marcada variación interanual y una sucesión de estadíos secos y húmedos en una larga serie de años.

Por lo tanto, este trabajo tiene como objetivo principal detectar las tendencias actuales de la precipitación en nuestra provincia durante el período 1955/2009. En el mismo se han empleado 18 puestos pluviométricos distribuidos por toda la provincia de manera de obtener información representativa. Como resultados parciales se observa una alta variación interanual que responde, según se encuentren al Oeste o al Este, al factor continental y al Fenómeno del Niño u Oscilación del sur, respectivamente.

También es posible establecer una variación Este-Oeste con respecto de la duración de los estadíos secos y húmedos detectados. En el Oriente provincial los estadíos húmedos son más prolongados y los secos más cortos mientras que, en el Occidente, los húmedos son más cortos al igual que los estadíos secos, no obstante se suceden algunas excepciones.

Palabras Claves: Variabilidad Interanual. Fluctuación Pluviométrica. Dinámica Espacial.

\section{Summary:}

The rainfall and temperature are together the elements of weather that arouses interest in the scientific community because definitely they influence the economic activities of the population.

The Precipitation, however, is characterized by highly variable making it difficult to establish a behavior pattern in time or space in a long series of years to help when making economic decisions and social policies.

In previous studies about the dynamics rainfall in the province of Chaco has detected an increasing rainfall gradient from west-east, a marked interannual variation and a sequence of dry and wet stages in a long series of years

Therefore, this study aims to identify trends leading to the precipitation in the province throughout the period 1955/2009. In the same has been used 18 rainfall positions distributed throughout the province in order to obtain representative data. In this study there is a high interannual variation respondent, as found in the west or east, the continental factor and the El Niño or Southern Oscillation, respectively.

It is also possible to establish an East-West variation with respect to the duration of wet and dry stages detected.

Publicado en formato digital: Prof. Claudia Gómez y Prof. María Emilia Pérez. LA VARIABILIDAD PLUVIOMÉTRICA EN LA PROVINCIA DEL CHACO 1955 Y 2009. Revista Geográfica Digital. IGUNNE. Facultad de Humanidades. UNNE. Año 8. No 16. Julio - Diciembre 2011. ISSN 1668-5180 Resistencia, Chaco. En: http://hum.unne.edu.ar/revistas/geoweb/default.htm 
In the East provincial wet stages are longer and shorter dry, while in the West, are shorter wet as dry stages, though some exceptions will occur.

Keywords: Interannual variability. Rainfall fluctuation. Space Dynamics. 


\section{"LA VARIABILIDAD PLUVIOMÉTRICA EN LA PROVINCIA DEL CHACO DURANTE EL PERÍODO 1955/2009".}

Prof. Gómez, Claudia Verónica (Dpto. de Geografía-Facultad de Humanidades-UNNE)

Prof. Pérez, María Emilia (Dpto. de Geografía-Facultad de Humanidades-UNNE)

\section{INTRODUCCIÓN:}

La precipitación conjuntamente con la temperatura son los elementos más importantes del clima y, por lo tanto, los más estudiados. Ambos son de interés por las consecuencias directas que ejercen en la población y sus actividades económicas.

La precipitación, a diferencia de la temperatura, posee como característica principal una alta variabilidad temporal y espacial. Esta variabilidad responde básicamente a la circulación general de la atmósfera y, tal como lo señalan Cuadrat y Pita (2000) "a las variaciones en latitud que experimentan los cinturones zonales de vientos a lo largo del año", esto determina, en consecuencia, no sólo diferentes regímenes pluviométricos sino también, en una larga serie de años, "Períodos" muy Húmedos y muy Secos.

Con respecto a esto último Cuadrat y Pita (2000) también señalan que "lo característico de la precipitación es su "variabilidad" espacial y temporal, y la posibilidad de que se alcancen valores extremos, tanto de abundancia como de escasez, que por sus consecuencias catastróficas y su impacto sobre la sociedad forman parte destacada de los llamados riesgos naturales". Como se puede apreciar, estas breves palabras, destacan la importancia del estudio de la dinámica pluviométrica para el desarrollo social y económico de la población.

El presente trabajo trata sobre la "variabilidad" temporal y espacial de las precipitaciones en la provincia del Chaco durante el período 1955/2009. Esta serie comprende 56 años de datos estadísticos mensuales. Sin embargo, se debe tener en cuenta que, según los estudios climáticos actuales, esta longitud temporal es considerada insuficiente para detectar todas las variaciones posibles en la dinámica pluviométrica.

El interés de este estudio en nuestra provincia, se fundamenta en lo expuesto anteriormente con respecto de "la posibilidad de alcanzar valores extremos de abundancia y escasez" de precipitaciones como consecuencia de la "variabilidad" que le es propia. En la provincia del Chaco más que una posibilidad es una realidad a la que generalmente estamos expuestos por las características intrínsecas del clima "Subtropical" que presenta nuestra región. De más está decir que, el tema es de particular importancia para la población, dado que la actividad económica en la provincia es fundamentalmente agrícola y ganadera cuya continuidad y desarrollo está sujeto a estas variaciones pluviométricas.

Sin embargo, a la población le preocupa más los períodos de escasez que los períodos de extrema precipitaciones puesto que estos últimos, según estos mismos autores, "son desastres de corta duración, pudiendo prolongarse desde unas horas a un mes, y tienen extensión espacial limitada a las márgenes de los ríos o a zonas más extensas cuando el territorio es llano o con mal drenaje", comparados con los períodos de sequías que es un fenómeno constante y con frecuencia de alcance regional.

Por otra parte, cabe decir que, este informe al margen del polémico Cambio Climático y de afirmaciones tales como "aumento en la frecuencia de sequías en determinadas zonas del planeta, en especial los países ubicados en las zonas subtropicales", se estudia a la dinámica pluviométrica como altamente variable pero asimismo estable, es decir, que los valores de precipitación "fluctúan" aleatoriamente entorno a un valor central. Entonces, tal como lo señala Cuadrat y Pita (2000), se realiza este estudio considerando al clima como "la 
generalización estadística de su comportamiento atmosférico, considerado éste como estable, como estacionario, aunque enormemente variable de unos momentos a otros".

En nuestro espacio de estudio se ha seleccionado 18 puestos pluviométricos, teniendo en cuenta tres aspectos esenciales: en primer lugar, la disponibilidad de datos, en segundo lugar, la longitud, homogeneidad y representatividad de la serie estadística y, en tercer lugar, su localización espacial, de manera de cubrir el mayor área posible y obtener información representativa. Los puestos pluviométricos seleccionados se encuentran consignados en la Tabla № 1 y localizados en el Mapa № 1.

\section{Tabla No 1: Puestos Pluviométricos de la Provincia del Chaco}

\begin{tabular}{|l|l|l|l|}
\hline Puestos Pluviométricos & \multicolumn{1}{|c|}{ Departamento } & \multicolumn{1}{|c|}{ Latitud } & Longitud \\
\hline Taco Pozo & Almirante Brown & $25^{\circ} 36^{\prime} 50^{\prime \prime}$ & $63^{\circ} 16^{\prime} 2^{\prime \prime}$ \\
\hline El Sauzalito & General Güemes & $24^{\circ} 25^{\prime} 25^{\prime \prime}$ & $61^{\circ} 40^{\prime} 55^{\prime \prime}$ \\
\hline M. Nueva Pompeya & General Güemes & $24^{\circ} 55^{\prime} 43^{\prime \prime}$ & $61^{\circ} 29^{\prime} 11^{\prime \prime}$ \\
\hline Juan José Castelli & General Güemes & $25^{\circ} 56^{\prime} 47^{\prime \prime}$ & $60^{\circ} 37^{\prime} 20^{\prime \prime}$ \\
\hline Los Frentones & Almirante Brown & $26^{\circ} 24^{\prime} 26^{\prime \prime}$ & $61^{\circ} 24^{\prime} 56^{\prime \prime}$ \\
\hline Concepción del Bermejo & Almirante Brown & $26^{\circ} 35^{\prime} 50^{\prime \prime}$ & $60^{\circ} 56^{\prime} 41^{\prime \prime}$ \\
\hline Gancedo & 12 de Octubre & $27^{\circ} 29^{\prime} 27^{\prime \prime}$ & $61^{\circ} 40^{\prime} 36^{\prime \prime}$ \\
\hline P. Roque Saenz Peña & Cte. Fernández & $26^{\circ} 46^{\prime} 57^{\prime \prime}$ & $60^{\circ} 26^{\prime} 26^{\prime \prime}$ \\
\hline Presidencia Roca & L. Gral. San Martín & $26^{\circ} 8^{\prime} 13^{\prime \prime}$ & $59^{\circ} 35^{\prime} 48^{\prime \prime}$ \\
\hline Chorotis & F.J. Sta. Ma. De Oro & $27^{\circ} 54^{\prime} 54^{\prime \prime}$ & $61^{\circ} 24^{\prime} 12^{\prime \prime}$ \\
\hline Coronel Du Graty & M. J. Luis Fontana & $27^{\circ} 41^{\prime} 3^{\prime \prime}$ & $60^{\circ} 54^{\prime} 7^{\prime \prime}$ \\
\hline Villa Berthet & San Lorenzo & $27^{\circ} 17^{\prime} 24^{\prime \prime}$ & $60^{\circ} 24^{\prime} 52^{\prime \prime}$ \\
\hline Pcia. de La Plaza & Pcia. de La Plaza & $27^{\circ} 0^{\prime} 3^{\prime \prime}$ & $59^{\circ} 50^{\prime} 45^{\prime \prime}$ \\
\hline General San Martín & L. Gral. San Martín & $26^{\circ} 32^{\prime} 17^{\prime \prime}$ & $59^{\circ} 20^{\prime} 26^{\prime \prime}$ \\
\hline La Sabana & Tapenagá & $27^{\circ} 52^{\prime} 21^{\prime \prime}$ & $59^{\circ} 56^{\prime} 16^{\prime \prime}$ \\
\hline Basail & San Fernando & $27^{\circ} 53^{\prime} 5^{\prime \prime}$ & $59^{\circ} 16^{\prime} 41^{\prime \prime}$ \\
\hline Resistencia & San Fernando & $27^{\circ} 26^{\prime} 23^{\prime \prime}$ & $59^{\circ} 0^{\prime} 0^{\prime \prime}$ \\
\hline General Vedia & Bermejo & $26^{\circ} 56^{\prime} 8^{\prime \prime}$ & $58^{\circ} 39^{\prime} 38^{\prime \prime}$ \\
\hline
\end{tabular}

El período estudiado comprende los años 1955 a 2009; los datos pluviométricos empleados se han recopilado del Boletín publicado por la Administración Provincial de Agua (A.P.A) denominado "Anuario de Precipitaciones Período 1956/2010" que se encuentra disponible en su página web http://www.chaco.gov.ar/apa.

Si bien la mayoría de las estaciones pluviométricas presenta la serie completa, cuatro de ellas, localizadas en el Occidente Provincial, poseen importantes lagunas, por ejemplo: Taco Pozo cuenta con datos desde 1974/2009, Nueva Pompeya desde 1978/2009 y El Sauzalito desde 1984/2009. Estas lagunas, hasta el momento, no pudieron ser rellenadas ya que no se cuenta con datos de estaciones pluviométricas cercanas que presenten la serie homogénea y completa del período de estudio. 


\section{Mapa № 1: Localización Geográfica}

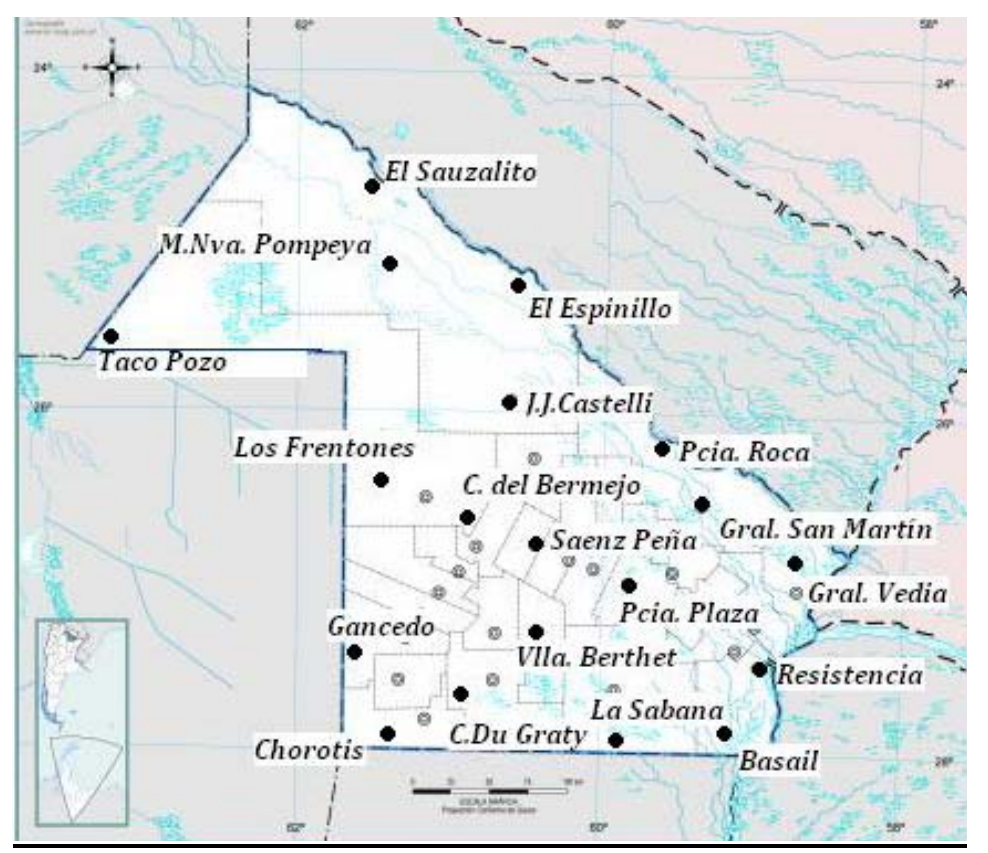

\section{OBJETIVOS Y MÉTODOS:}

Los objetivos que se han propuesto alcanzar y que han estructurado la realización de este trabajo son básicamente dos:

1. Analizar los datos pluviométricos anuales y quinquenales durante el período 1955/2009 y estudiar el comportamiento temporal y espacial de las precipitaciones.

2. Identificar la "variabilidad" temporo-espacial de las mismas y detectar tendencias actuales.

En cuanto a los pasos metodológicos que se han ejecutado, los mismos consistieron en:

1. Recopilación de datos estadísticos y búsqueda bibliográfica.

2. Confección de una base de datos estadísticos.

3. Homogeneización de las series y rellenos de lagunas.

4. Tratamiento y normalización de los datos estadísticos: cálculos de promedios, desviación y coeficiente de variación.

5. Representación gráfica.

6. Realización del informe y divulgación de las conclusiones.

\section{DESARROLLO:}

Este trabajo está orientado al estudio de la variabilidad pluviométrica y la detección de tendencias actuales de la precipitación en la Provincia del Chaco durante el período 1955/2009. Con anterioridad se ha tratado el régimen pluviométrico de algunas de las localidades aquí estudiadas y se analizó en detalle su comportamiento diferencial y las causas que lo originan, según se localicen en el sector Oriental u Occidental. Entre las conclusiones a las que se arribaron en ese estudio podemos mencionar, la detección, tanto en el sector Occidental como el Oriental, de "períodos" húmedos y secos sucesivos, una

Publicado en formato digital: Prof. Claudia Gómez y Prof. María Emilia Pérez. LA VARIABILIDAD PLUVIOMÉTRICA EN LA PROVINCIA DEL CHACO 1955 Y 2009. Revista Geográfica Digital. IGUNNE. Facultad de Humanidades. UNNE. Año 8. No 16. Julio - Diciembre 2011. ISSN 1668-5180 Resistencia, Chaco. En: http://hum.unne.edu.ar/revistas/geoweb/default.htm 
clara disminución de los montos pluviométricos en el primero de ellos y una marcada variación interanual durante el período de estudio 1957/2005.

El Gráfico No 1 muestra la variación anual de las precipitaciones durante el período de estudio. Cabe aclarar que las localidades representadas han sido distribuidas según su localización espacial, en el Oeste, Centro y Este del territorio provincial, distribución que se ajusta además, al comportamiento pluviométrico descripto, que nos permite agrupar las localidades en tres clases o grupos relativamente diferenciados que facilitan su explicación. Ver Gráfico 1. Figuras 1A, 1B, 1C.

A simple vista se puede detectar una marcada variación interanual, es decir, una sucesión entre años más húmedos y otros secos en todos los puestos pluviométricos. Sin embargo, es necesario aclarar que, las causas de esta variación difieren según se trate del sector Occidental u Oriental de la Provincia del Chaco.

En el primer grupo (1 A) los puestos pluviométricos de El Sauzalito, Misión Nueva Pompeya (con excepción del año 1996 con 1186 mm) y Taco Pozo presentan montos inferiores a los $1000 \mathrm{~mm}$ de precipitación.

En el resto de las localidades se puede observar que, si bien la mayoría de los montos pluviométricos de la serie trabajada se encuentra por debajo de los $1000 \mathrm{~mm}$, denotan un aumento sensible de años que superan este valor y en el caso de Concepción del Bermejo, durante los años 1959 (con 1549 mm) y 2002 (con 1635 mm), pueden superar, incluso, los $1500 \mathrm{~mm}$ aunque, cabe decir, muy ocasionalmente.

Esto último pone de relieve la variabilidad interanual mencionada anteriormente, la cual responde a las características del clima subtropical y al factor continental. En el Occidente Chaqueño lo normal son montos pluviométricos inferiores a los $1000 \mathrm{~mm}$., por lo tanto la variabilidad interanual se genera por aquellos montos que superan notablemente este valor y que se deben a lluvias abundantes caídas excepcionalmente.

En los gráficos se ha incluido también, la tendencia de los montos anuales calculada a partir de la Media Móvil, representada con una línea de color rojo. El método consiste en calcular los valores medios para períodos consecutivos, en este caso particular de 6 años.

Esta curva destaca, en toda las estaciones trabajadas, "oscilaciones" positivas o negativas que indican el desarrollo de "períodos" más húmedos alternados con otros más secos en el área de estudio. Se han podido detectar hasta tres "períodos" húmedos en la estación de El Sauzalito, uno de los cuales está completo y comprende desde 1990 hasta 2002, como así también dos períodos secos alternados.

En Misión Nueva Pompeya también se puede observar dos períodos húmedos y dos períodos secos bien marcados, los cuales describen similar comportamiento al anterior con la diferencia de que el período húmedo completo se extiende desde 1990 hasta 2005 aproximadamente.

Una situación inversa se presenta en Taco Pozo donde también se puede advertir dos "períodos" húmedos bien marcados separados por un período seco extenso, comparado con las estaciones anteriores, y que se extiende desde 1985 hasta 1999.

Las estaciones de J.J. Castelli, Gancedo y Chorotis presentan tres períodos húmedos alternados con dos períodos secos. Cabe aclarar que los mismos describen oscilaciones muy suavizadas y se desarrollan en períodos de tiempos similares.

Concepción del Bermejo, al igual que Taco pozo, no parece ajustarse a la tendencia general de las estaciones localizadas en el Occidente chaqueño, ya que presenta dos períodos húmedos bien marcados intercalados con un período seco. El período húmedo que

Publicado en formato digital: Prof. Claudia Gómez y Prof. María Emilia Pérez. LA VARIABILIDAD PLUVIOMÉTRICA EN LA PROVINCIA DEL CHACO 1955 Y 2009. Revista Geográfica Digital. IGUNNE. Facultad de Humanidades. UNNE. Año 8. No 16. Julio - Diciembre 2011. ISSN 1668-5180 Resistencia, Chaco. En: http://hum.unne.edu.ar/revistas/geoweb/default.htm 
se extiende desde 1972 hasta 2007 es comparativamente más extenso de lo que le correspondería a su localización, sin embargo, describe un comportamiento parecido a las estaciones analizadas a continuación.

Gráfico No 1 Figura 1.A. Variación Anual de las Precipitaciones durante el Período 1955/2009.

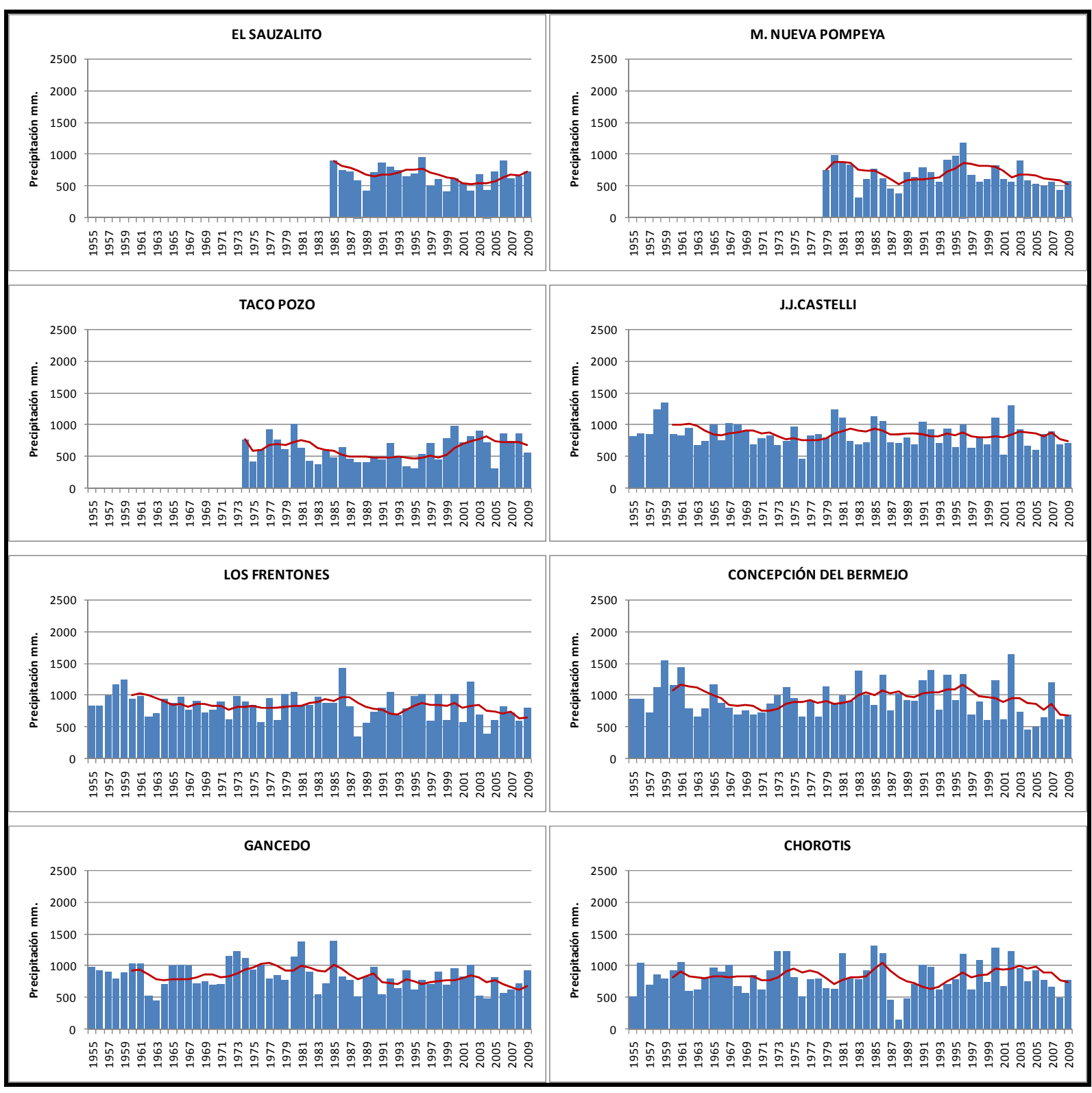

Fuente: datos proporcionados por la APA (Administración Provincial del Agua)

En las estaciones del grupo (1 B), localizadas en el centro provincial, se puede observar que, se suceden con mayor frecuencia los años con montos de precipitación superiores a los 1000 mm., e incluso en Presidencia Roca (en 1986 presenta 1903 mm), en Sáenz Peña (en 1996 con 1525 mm) y Coronel Du Graty (en 1998 con 1635 mm) superan los 1500 mm. Esto denota un aumento en los valores de precipitación en comparación con las estaciones anteriores y pone en evidencia el gradiente pluviométrico Oeste-Este.

Publicado en formato digital: Prof. Claudia Gómez y Prof. María Emilia Pérez. LA VARIABILIDAD PLUVIOMÉTRICA EN LA PROVINCIA DEL CHACO 1955 Y 2009. Revista Geográfica Digital. IGUNNE. Facultad de Humanidades. UNNE. Año 8. No 16. Julio - Diciembre 2011. ISSN 1668-5180 Resistencia, Chaco. En: http://hum.unne.edu.ar/revistas/geoweb/default.htm 
Gráfico No 1 Figura 1.B. Variación Anual de las Precipitaciones durante el Período 1955/2009.

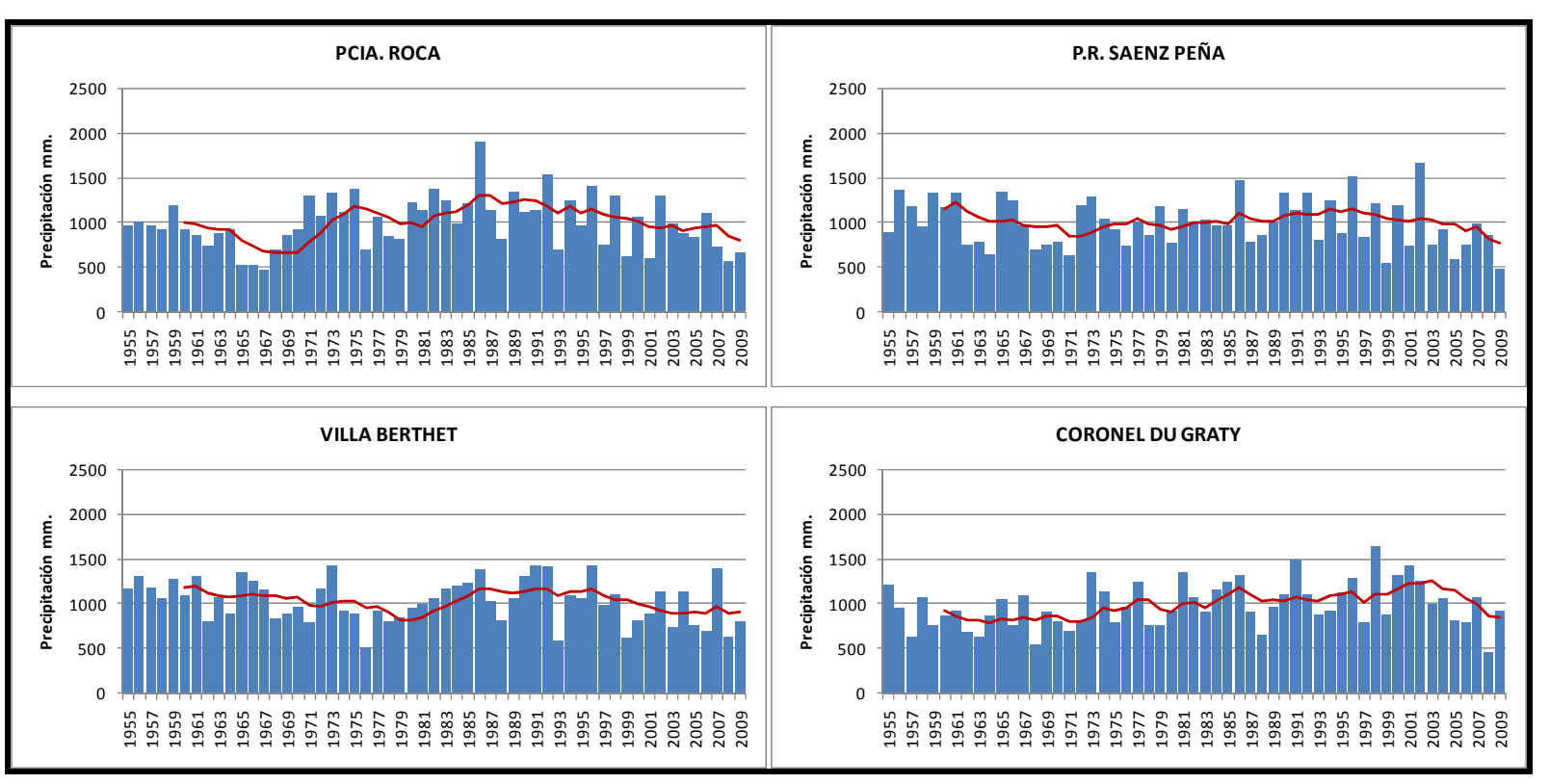

Fuente: datos proporcionados por la APA (Administración Provincial del Agua)

Con respecto a la curva de promedios móviles o suavizados, se observa que, los puestos de Sáenz Peña y Du Graty describen, casi imperceptiblemente, un período seco y un período húmedo. Estas fluctuaciones se presentan con un leve desfasaje, es decir, que en Sáenz Peña el período seco se desarrolla desde 1962 hasta 1989 y en Du Graty desde 1957 hasta 1980.

Las curvas de tendencia de Presidencia Roca y Villa Berthet, por su parte, poseen un comportamiento más acentuado detectándose perfectamente un período seco, relativamente corto y desfasado, alternado con períodos húmedos más extensos.

En cuanto a las localidades del último grupo (1.C), se advierte claramente que, a medida que nos desplazamos hacia el Oriente, los montos pluviométricos aumentan, y oscilan entre parámetros de 1000 y 2000 mm. de precipitación, particularmente en las estaciones de General Vedia, Resistencia y Basail, no obstante se puede apreciar que en algunos casos se supera este valor como en los años 1973, 1996 y 1998 en Vedia; 1973, 1986 y 1996 en Resistencia; 1986, 1998 y 2000 en Basail.

Lo característico de este grupo, además de presentar con mayor frecuencia montos anuales superiores a los $1000 \mathrm{~mm}$, es la presencia de tres períodos húmedos intercalados con períodos secos bien marcados. El último de ellos es el más definido, comportamiento que responde al ciclo más lluvioso de fines del siglo XX en el cono sur de América del Sur (Ropelewski and Halpert, 1987; Nicholls and Wong, 1990; Minetti y Vargas, 1997; Pérez, 2006 entre otros).

No obstante, se detectan algunas diferencias en las localidades de Gral. San Martín y Presidencia Plaza, cuyo comportamiento se asemeja más a las estaciones del grupo $1 \mathrm{~B}$, posiblemente por su mayor cercanía a las mismas.

En este grupo llama la atención, además, la marcada variabilidad interanual que presentan las precipitaciones. Según los expertos del clima la variabilidad interanual es alta en regiones semiáridas y baja en los climas húmedos, es decir, que esta variabilidad aumenta a medida que el monto anual de la lluvia decrece (Conrad, 1941). Sin embargo y tal como puede observarse en nuestro caso, esta "regla" no se aplica, debido a que la lluvia

Publicado en formato digital: Prof. Claudia Gómez y Prof. María Emilia Pérez. LA VARIABILIDAD PLUVIOMÉTRICA EN LA PROVINCIA DEL CHACO 1955 Y 2009. Revista Geográfica Digital. IGUNNE. Facultad de Humanidades. UNNE. Año 8. No 16. Julio - Diciembre 2011. ISSN 1668-5180 Resistencia, Chaco. En: http://hum.unne.edu.ar/revistas/geoweb/default.htm 
anual responde a la influencia del Fenómeno del Niño u Oscilación del Sur que provoca precipitaciones aún más abundantes que las registradas normalmente (Nicholls, 1988; Nicholls and Wong, 1990; Dewar y Wallis 1999, entre otros autores). Sus estudios pusieron de manifiesto que la variabilidad de la lluvia anual aumenta a medida que 1) la precipitación media anual disminuye, 2) cuando la latitud disminuye y 3) en las regiones tropicales y cerca de las regiones tropicales cuando la influencia de la Oscilación del Sur aumenta

Gráfico No 1 Figura 1.C. Variación Anual de las Precipitaciones durante el Período 1955/2009.

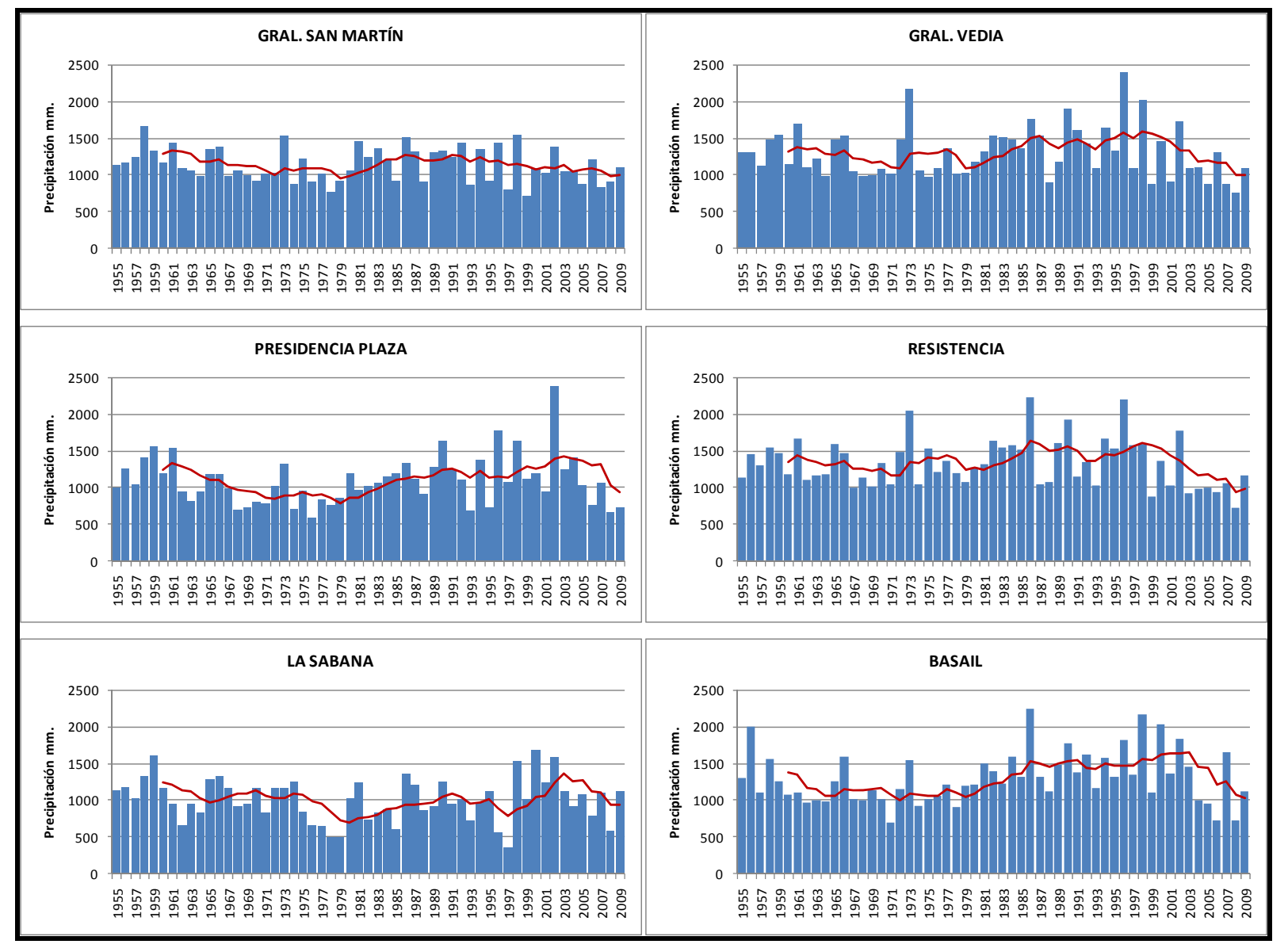

Fuente: datos proporcionados por la APA (Administración Provincial del Agua)

Lo analizado hasta el momento destaca que en toda las estaciones trabajadas no se evidencia una tendencia positiva o negativa preponderante hacia el aumento o disminución continua de los montos, sino que lo habitual son "oscilaciones" ascendente y descendentes destacando la presencia de "períodos" más húmedos y otros más secos en el área de estudio.

La Figura $\mathbf{N}^{\circ} 2$ representa las desviaciones de los montos anuales con respecto de la media del período de estudio. Estas desviaciones están expresadas en porcentaje para facilitar la comparación entre las estaciones pluviométricas. Asimismo cabe aclarar que las mismas se disponen respetando la distribución anterior.

Lo que puede observarse a simple vista es que, en todas las estaciones de estudio, es frecuente la sucesión de años húmedos o muy húmedos con años secos o muy secos. También es importante destacar que, si tenemos en cuenta la característica altamente variable de las precipitaciones, es difícil detectar un patrón de comportamiento en las

Publicado en formato digital: Prof. Claudia Gómez y Prof. María Emilia Pérez. LA VARIABILIDAD PLUVIOMÉTRICA EN LA PROVINCIA DEL CHACO 1955 Y 2009. Revista Geográfica Digital. IGUNNE. Facultad de Humanidades. UNNE. Año 8. No 16. Julio - Diciembre 2011. ISSN 1668-5180 Resistencia, Chaco. En: http://hum.unne.edu.ar/revistas/geoweb/default.htm 
mismas. En general, las desviaciones positivas y negativas desde la normal oscilan en la mayoría de los años entre el 58 y $75 \%$ para las desviaciones positivas y entre -35 y $-50 \%$ para las negativas.

\section{Gráfico № 2 Figura 2 A: Desviación en Porcentaje (\%) de los Montos desde la Normal}

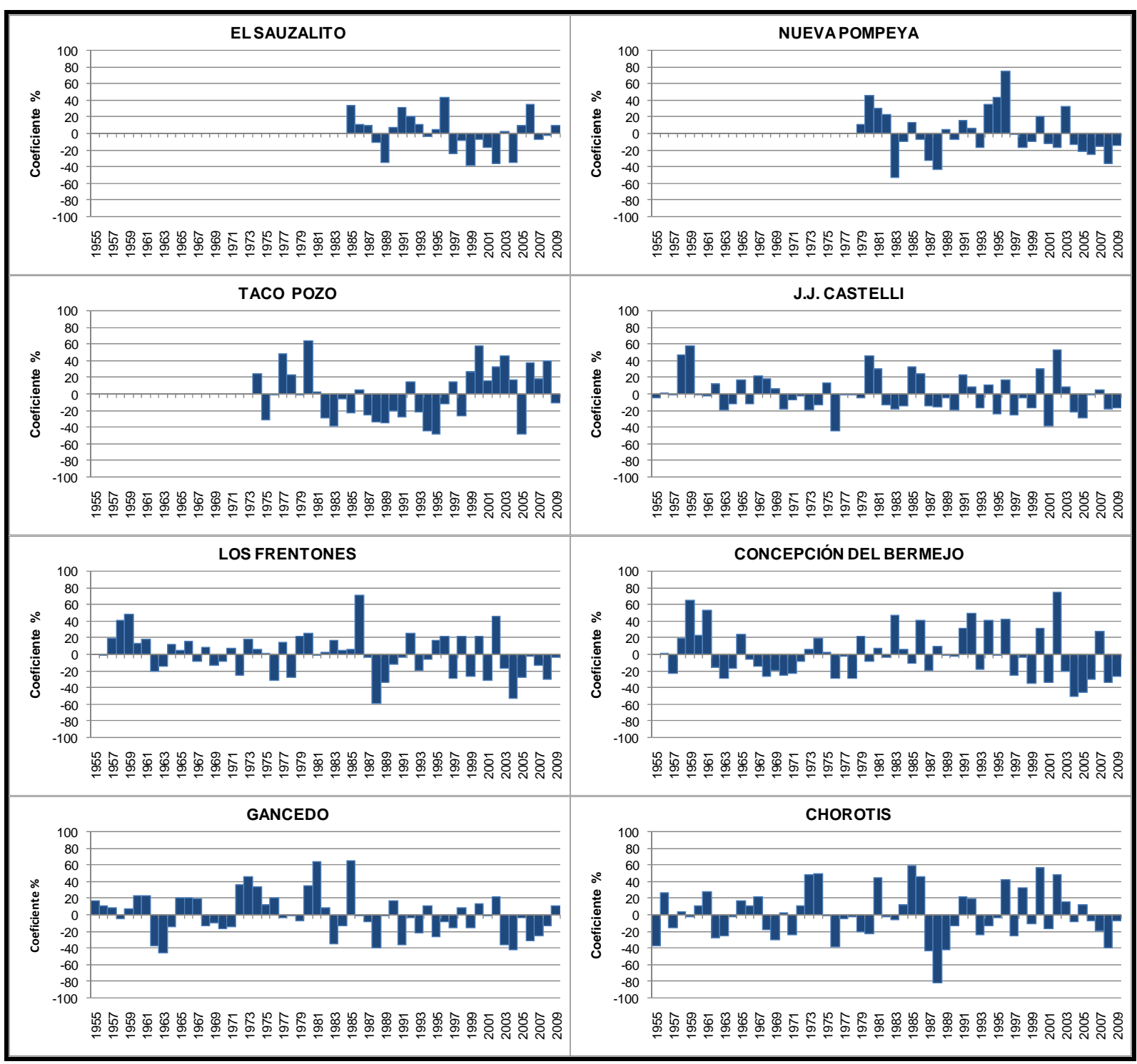

Fuente: datos proporcionados por la APA (Administración Provincial del Agua)

No obstante se pueden advertir situaciones especiales que superan ampliamente los límites de las desviaciones positivas. Por ejemplo, en las estaciones del grupo 2 A, Nueva Pompeya (en el año 1996), Taco Pozo (1980 y 2000), Los Frentones (1986), Concepción del Bermejo (1959 y 2002) y en Gancedo (1981 y 1985). Un dato particular a tener en cuenta, en el período trabajado, es que ninguna de estas estaciones presenta una disminución de sus montos que superen el -60 \%, a excepción del año 1988 en Chorotis (-82\%), que puede deberse a un subregistro ya que las localidades cercanas no muestran esta característica.

Por otra parte, es necesario destacar que en los últimos 7 u 8 años se registran desviaciones negativas en la mayoría de estas estaciones, a excepción de la localidad de Taco Pozo que sigue un comportamiento inverso, sin embargo, cabe decir que en algunas

Publicado en formato digital: Prof. Claudia Gómez y Prof. María Emilia Pérez. LA VARIABILIDAD PLUVIOMÉTRICA EN LA PROVINCIA DEL CHACO 1955 Y 2009. Revista Geográfica Digital. IGUNNE. Facultad de Humanidades. UNNE. Año 8. NN 16. Julio - Diciembre 2011. ISSN 1668-5180 Resistencia, Chaco. En: http://hum.unne.edu.ar/revistas/geoweb/default.htm 


\begin{tabular}{|l|l|}
\hline Revista Geográfica Digital. IGUNNE. Facultad de Humanidades. UNNE. Año 8. № 16. Julio- Diciembre 2011. \\
ISSN 1668-5180 Resistencia, Chaco
\end{tabular}

localidades este período seco se alivia por uno, dos y hasta tres años exiguamente húmedos.

Las desviaciones de los puestos pluviométricos del grupo 2 B oscilan entre los parámetros de 38 y $-55 \%$. Se puede detectar, además, que en Presidencia Roca se alcanza el $90 \%$ del monto con respecto a la normal (en el año 1986) y en Saenz Peña y Du Graty se excede un $60 \%$ (en 2002 y 1998 respectivamente). Las desviaciones negativas comprenden valores de alrededor de $-50 \%$.

Gráfico № 2 Figura 2 B: Desviación en Porcentaje (\%) de los Montos desde la Normal

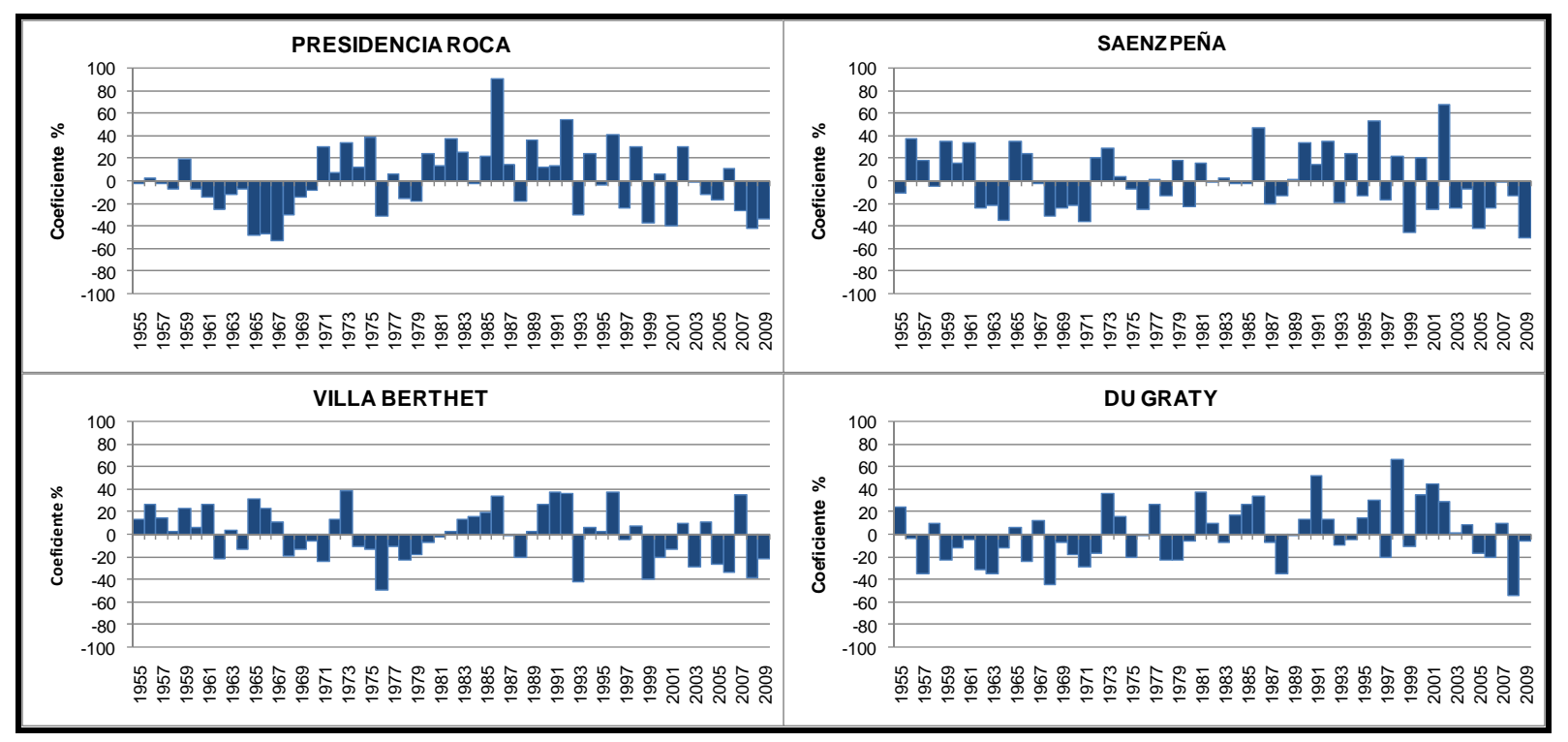

Fuente: datos proporcionados por la APA (Administración Provincial del Agua)

Las estaciones del último grupo $(2 \mathrm{C})$ evidencian un comportamiento muy similar al anterior con años que superan ampliamente el $60 \%$, como por ejemplo, los registrados en Pcia. De la Plaza (119\% en el año 2002) y Gral Vedia (84\% en 1996)

Cabe recalcar que en todos los puestos pluviométricos aquí estudiados se evidencia que en los últimos 7 u 8 años los montos de precipitación se mantienen por debajo de la media del período aunque escasamente interrumpidos por uno o dos años relativamente húmedos.

Publicado en formato digital: Prof. Claudia Gómez y Prof. María Emilia Pérez. LA VARIABILIDAD PLUVIOMÉTRICA EN LA PROVINCIA DEL CHACO 1955 Y 2009. Revista Geográfica Digital. IGUNNE. Facultad de Humanidades. UNNE. Año 8. N N 16. Julio - Diciembre 2011. ISSN 1668-5180 Resistencia, Chaco. En: http://hum.unne.edu.ar/revistas/geoweb/default.htm 


\begin{tabular}{|l|l|}
\hline Revista Geográfica Digital. IGUNNE. Facultad de Humanidades. UNNE. Año 8. № 16. Julio- Diciembre 2011. \\
ISSN 1668-5180 Resistencia, Chaco
\end{tabular}

Gráfico № 2 Figura 2 C: Desviación en Porcentaje (\%) de los Montos desde la Normal

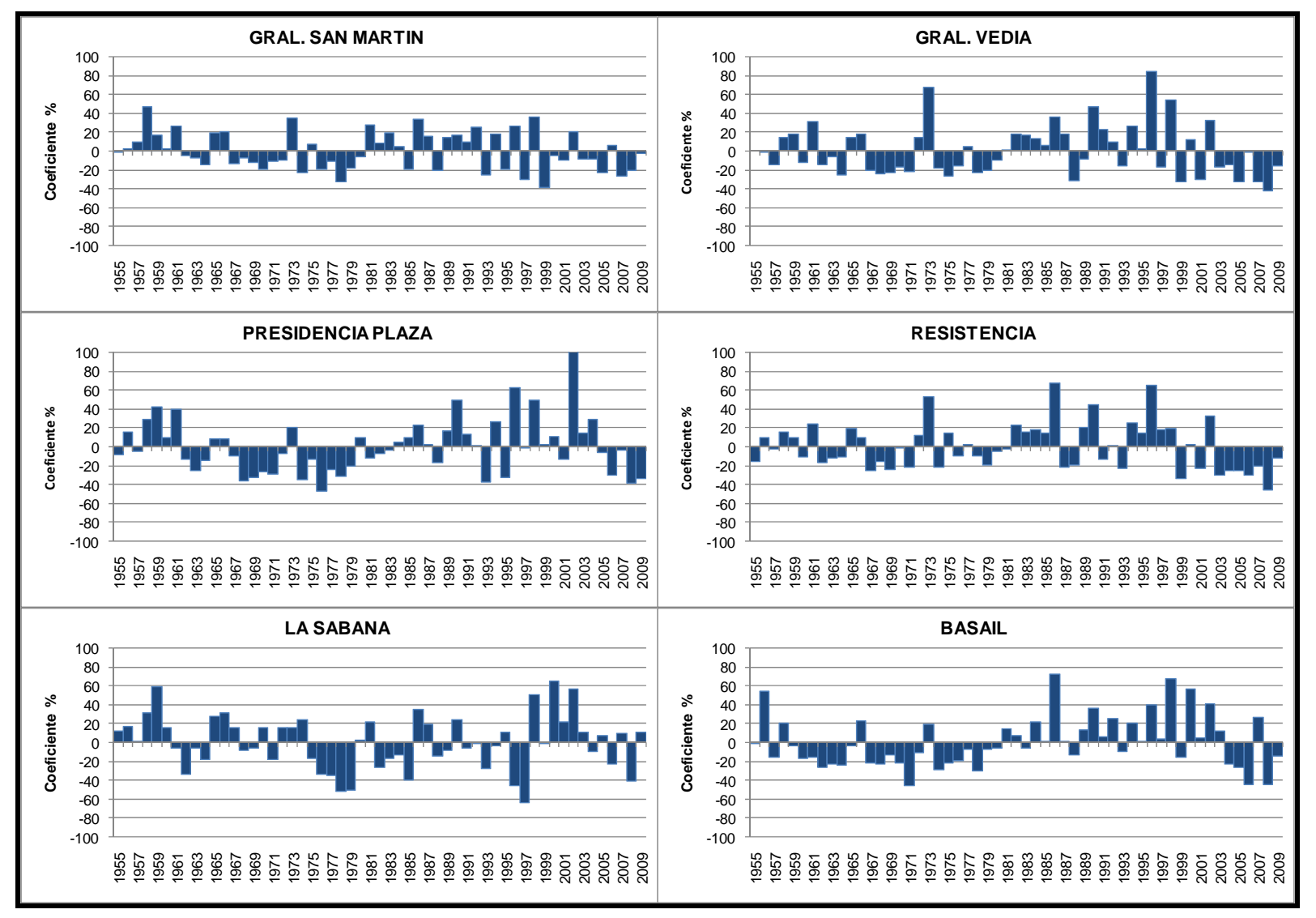

Fuente: datos proporcionados por la APA (Administración Provincial del Agua)

Otro de los análisis efectuados en este trabajo es el comportamiento de la precipitación por Quinquenios cuyos promedios se consignan en la Tabla № 2 , valores que corroboran lo expuesto anteriormente con respecto de los períodos húmedos y secos.

Tabla No 2: Montos Medios de Precipitación Anual por Quinquenio (mm.)

\begin{tabular}{|c|c|c|c|c|c|c|c|c|c|c|c|}
\hline & $1955 / 59$ & $1960 / 64$ & $1965 / 69$ & $1970 / 74$ & $1975 / 79$ & $1980 / 84$ & $1985 / 89$ & $1990 / 94$ & $1995 / 99$ & $2000 / 04$ & $2005 / 09$ \\
\hline Taco Pozo & $S / D$ & S/D & $S / D$ & 438 & 670 & 615 & 484 & 499 & 566 & 834 & 669 \\
\hline El Sauzalito & S/D & S/D & S/D & S/D & S/D & $\mathrm{S} / \mathrm{D}$ & 679 & 754 & 637 & 542 & 724 \\
\hline M. Nva. Pompeya & S/D & S/D & S/D & S/D & 747 & 728 & 590 & 722 & 799 & 693 & 523 \\
\hline J.J. Castelli & 1025 & 811 & 938 & 749 & 789 & 903 & 888 & 863 & 758 & 904 & 751 \\
\hline Los Frentones & 939 & 851 & 850 & 832 & 799 & 918 & 805 & 811 & 845 & 781 & 710 \\
\hline Conc. Del Bermejo & 1056 & 969 & 862 & 880 & 870 & 1031 & 975 & 1126 & 894 & 939 & 704 \\
\hline Gancedo & 905 & 754 & 904 & 983 & 879 & 939 & 866 & 783 & 743 & 765 & 736 \\
\hline Chorotis & 789 & 802 & 832 & 972 & 717 & 872 & 727 & 812 & 888 & 987 & 729 \\
\hline Coronel Du Graty & 929 & 799 & 872 & 961 & 902 & 1082 & 1020 & 1107 & 1142 & 1215 & 810 \\
\hline Villa Berthet & 1199 & 1036 & 1099 & 1056 & 797 & 1078 & 1105 & 1164 & 1038 & 949 & 857 \\
\hline P.R. Saenz Peña & 1147 & 942 & 1003 & 992 & 946 & 983 & 1025 & 1175 & 998 & 1062 & 739 \\
\hline Pcia. Roca & 1018 & 867 & 617 & 1151 & 961 & 1196 & 1287 & 1147 & 1012 & 968 & 784 \\
\hline Gral. San Martín & 1315 & 1149 & 1157 & 1078 & 971 & 1266 & 1197 & 1246 & 1083 & 1117 & 990 \\
\hline Pcia. De la Plaza & 1254 & 1087 & 958 & 928 & 797 & 1077 & 1169 & 1209 & 1270 & 1439 & 848 \\
\hline La Sabana & 1261 & 916 & 1136 & 1118 & 635 & 949 & 997 & 988 & 917 & 1310 & 940 \\
\hline Basail & 1228 & 1028 & 1200 & 1071 & 1079 & 1384 & 1497 & 1506 & 1554 & 1537 & 1034 \\
\hline Resistencia & 1381 & 1263 & 1240 & 1390 & 1275 & 1467 & 1496 & 1428 & 1557 & 1217 & 980 \\
\hline Gral. Vedia & 1094 & 1233 & 1209 & 1365 & 1095 & 1408 & 1353 & 1536 & 1542 & 1256 & 985 \\
\hline \multicolumn{4}{|c|}{ Valor inexacto } & & & & & & & & \\
\hline
\end{tabular}

Fuente: datos proporcionados por la APA (Administración Provincial del Agua)

En el Gráfico $\mathbf{N}^{\circ} \mathbf{3}$ se puede observar el comportamiento de las precipitaciones para cada una de las estaciones pluviométricas según quinquenio. Este describe una dinámica

Publicado en formato digital: Prof. Claudia Gómez y Prof. María Emilia Pérez. LA VARIABILIDAD PLUVIOMÉTRICA EN LA PROVINCIA DEL CHACO 1955 Y 2009. Revista Geográfica Digital. IGUNNE. Facultad de Humanidades. UNNE. Año 8. No 16. Julio - Diciembre 2011. ISSN 1668-5180 Resistencia, Chaco. En: http://hum.unne.edu.ar/revistas/geoweb/default.htm 
relativamente uniforme durante los tres primeros quinquenios 1955/59, 1960/64 y 1965/69 en todas las estaciones y, desde 1970/74 hasta 2000/04, se evidencia un comportamiento diferencial en los mismos. En el último período 2005/2009 se puede advertir una tendencia nuevamente uniforme en todas las localidades trabajadas.

Cabe destacar que en el quinquenio 1955/59 los valores, en la mayoría de las estaciones, superan los $800 \mathrm{~mm}$. a excepción de Taco Pozo, El Sauzalito y Nueva Pompeya (que no cuentan con datos), comparados al período 2005/09 cuyos valores disminuyen y en muchos casos son inferiores a los $800 \mathrm{~mm}$. como en Taco Pozo, El Sauzalito, Nueva Pompeya, J.J. Castelli, Los Frentones, Concepción del Bermejo, Gancedo, Chorotis, Saenz Peña y Presidencia Roca.

Por el contrario el comportamiento que se observa en los quinquenios comprendidos desde 1970/74 hasta 2000/04 pueden responder a períodos húmedos o relativamente húmedos con un comportamiento diferencial según la localización geográfica de las estaciones.

\section{Gráfico № 3: Montos Medios de Precipitación Anual por Quinquenio (mm.)}

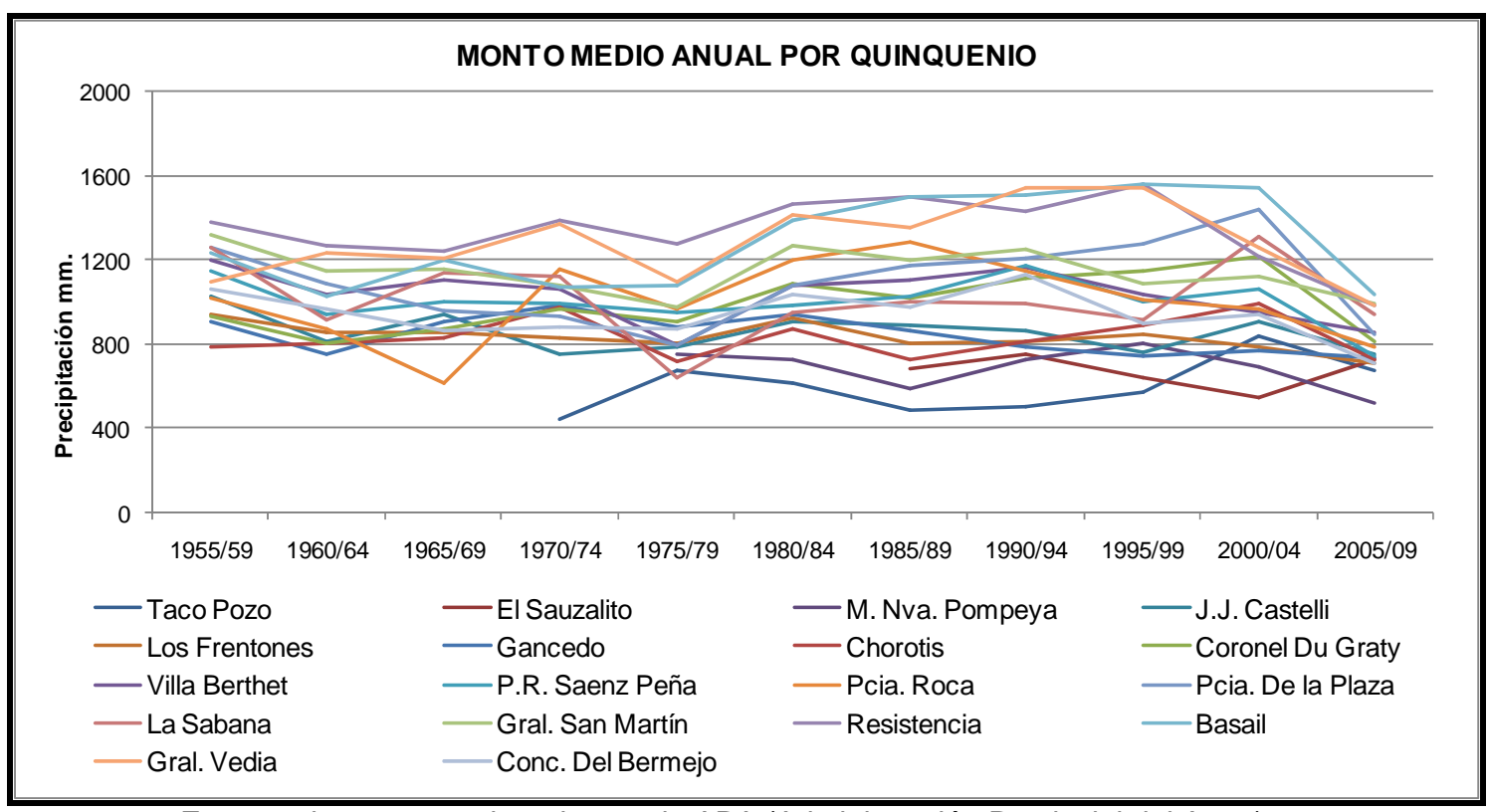

Fuente: datos proporcionados por la APA (Administración Provincial del Agua)

Se ha efectuado, además, un estudio del comportamiento de las precipitaciones medias mensuales por quinquenio, el cual se puede observar en el Gráfico $\mathbf{N}^{\circ} \mathbf{4}$. En este es posible detectar claramente la estación seca y húmeda estacional para cada uno de las localidades.

Asimismo se evidencia una cierta uniformidad en la media mensual quinquenal durante los períodos 1965/69 (con excepción de Los Frentones, Villa Berthet, Saenz Peña, San Martín, Resistencia y Vedia), 1970/74 Con excepción de Gancedo, Du Graty, Villa Berthet, La Sabana y Vedia) y 2005/2009 cuyos valores oscilan por debajo de los 200 mm. asimismo, otra característica a destacar, es que los máximos pluviométricos se registran generalmente en los meses de Enero-Febrero, Enero-Marzo, Noviembre-Diciembre-Enero, respectivamente.

Por el contrario, el resto del período, muestra un comportamiento diferencial que, en los puestos del Oriente y durante la estación húmeda, supera el valor de $200 \mathrm{~mm}$, registrándose los máximos pluviométricos en los meses de Marzo-Abril o Septiembre-Octubre-Noviembre.

Publicado en formato digital: Prof. Claudia Gómez y Prof. María Emilia Pérez. LA VARIABILIDAD PLUVIOMÉTRICA EN LA PROVINCIA DEL CHACO 1955 Y 2009. Revista Geográfica Digital. IGUNNE. Facultad de Humanidades. UNNE. Año 8. № 16. Julio - Diciembre 2011. ISSN 1668-5180 Resistencia, Chaco. En: http://hum.unne.edu.ar/revistas/geoweb/default.htm 


\begin{tabular}{|l|l|}
\hline Revista Geográfica Digital. IGUNNE. Facultad de Humanidades. UNNE. Año 8. № 16. Julio- Diciembre 2011. \\
ISSN 1668-5180 Resistencia, Chaco
\end{tabular}

Se puede especular que, durante los períodos 1965/69, 1970/74 y 2005/2009 lo característico es una disminución en los valores pluviométricos en la mayoría de las estaciones estudiadas que da cuenta de un período seco de alcance regional.

Gráfico № 4: Precipitación Media Mensual por Quinquenio (mm).

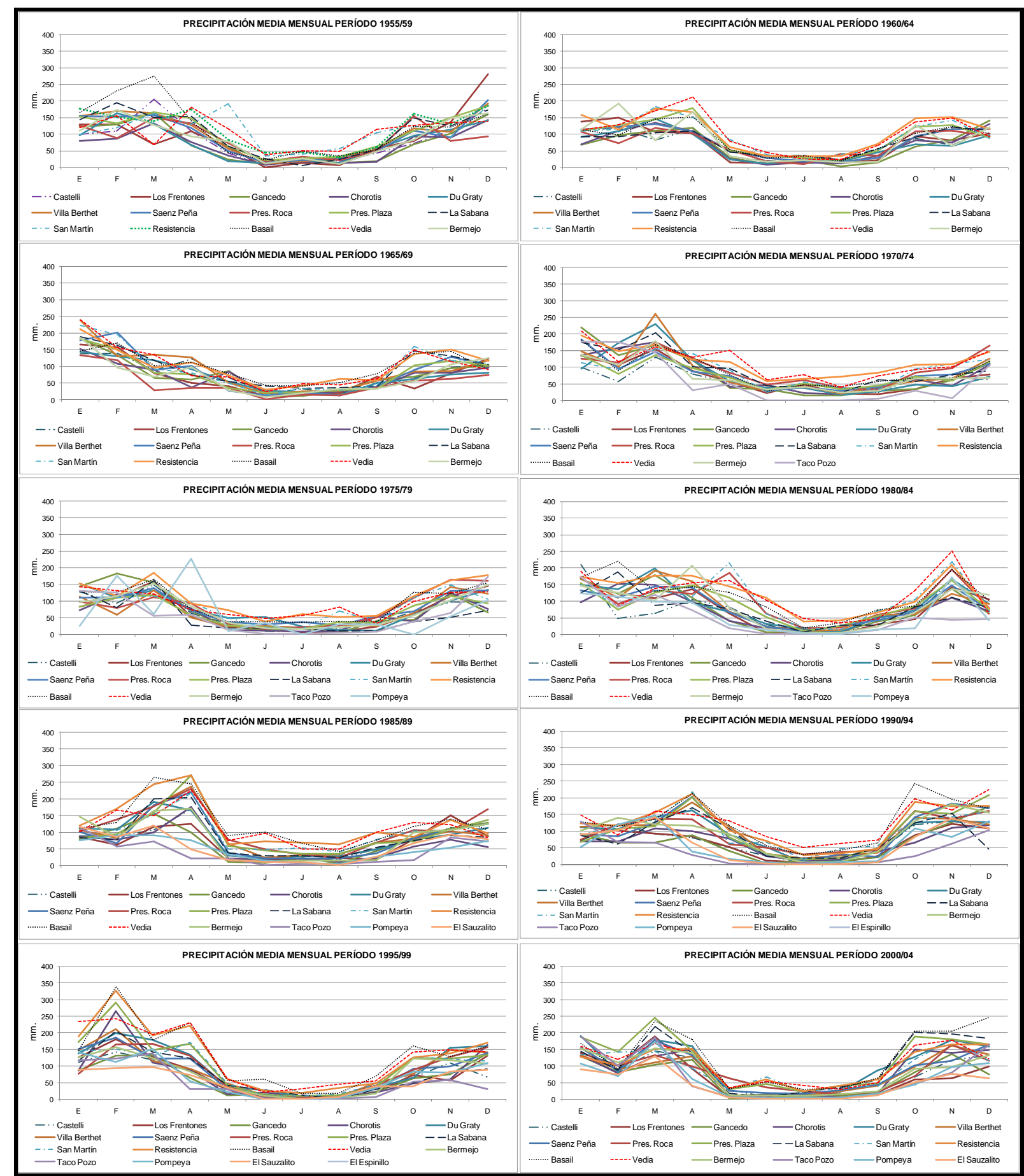

Fuente: datos proporcionados por la APA (Administración Provincial del Agua)

Publicado en formato digital: Prof. Claudia Gómez y Prof. María Emilia Pérez. LA VARIABILIDAD PLUVIOMÉTRICA EN LA PROVINCIA DEL CHACO 1955 Y 2009. Revista Geográfica Digital. IGUNNE. Facultad de Humanidades. UNNE. Año 8. No 16. Julio - Diciembre 2011. ISSN 1668-5180 Resistencia, Chaco. En: http://hum.unne.edu.ar/revistas/geoweb/default.htm 


\section{Continuación}

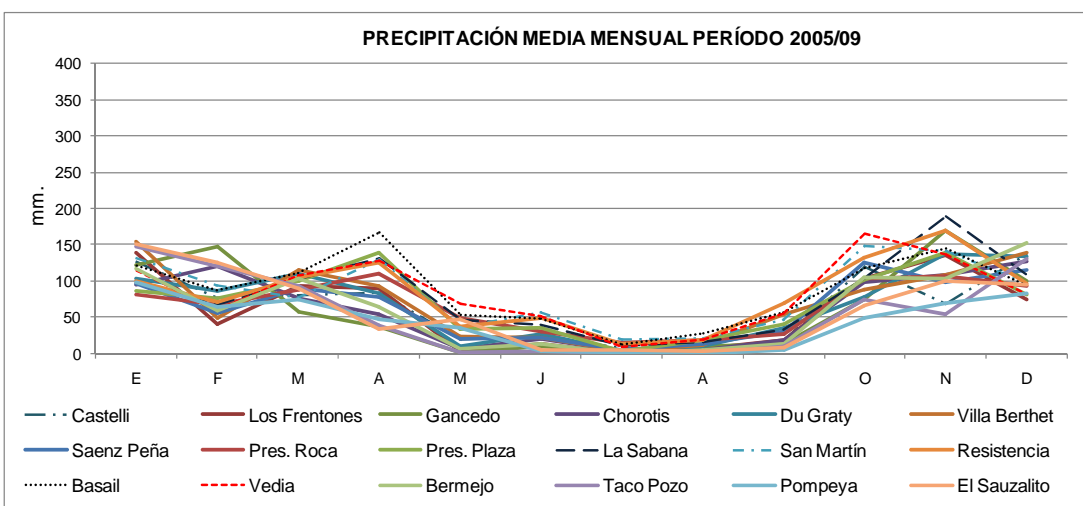

Fuente: datos proporcionados por la APA (Administración Provincial del Agua)

\section{CONCLUSIÓNES:}

Como se ha podido advertir, la Precipitación es un elemento del clima enormemente variable y, dado esta característica, es difícil establecer un patrón en su comportamiento temporal y espacial.

La dinámica pluviométrica temporal pone de manifiesto, en el período 1955/2009, una variación interanual marcada en las localidades del Occidente, Centro y Oriente chaqueño, las cuales responden a causas diferentes. Asimismo se destaca, en todas ellas, oscilaciones positivas y negativas alrededor de la media que en los casos analizados pueden presentarse relativamente desfasados y, como Taco Pozo, con un comportamiento inverso.

En cuanto a la dinámica espacial cabe señalar el aumento de los Montos pluviométricos desde el Occidente hacia el Oriente, es decir, un gradiente Oeste-Este. Además se ha observado en la serie trabajada que, en apariencia, existe un gradiente que aumenta de Este a Oeste respecto de las fluctuaciones positivas y negativas detectadas. Sin embargo, esto se debe a que, en el Este provincial los períodos secos son más cortos y los húmedos más largos en comparación con el Oeste en donde ambos se presentan más cortos y, aproximadamente, de similar duración.

Otro aspecto a destacar es que, en la Provincia del Chaco, las precipitaciones parecen seguir una tendencia actual generalizada. Esta consiste en una disminución de los montos pluviométricos durante los últimos 7 u 8 años con respecto a la normal, lo que indicaría que, actualmente nos encontramos en el interludio de una anomalía negativa cuya duración puede variar según se encuentre al Oeste o al Este de la provincia.

De lo desarrollado hasta el momento, y observando la variabilidad que presenta la precipitación, cabría preguntarse porque algunos investigadores tienden a fomentar e instaurar el denominado Cambio Climático.

Al parecer la respuesta tiene que ver con la indefinición del término "Cambio Climático" y el reconocimiento del mismo por la comunidad científica, ya que se utiliza una definición bastante imprecisa, entendiendo por Cambio Climático "como un término general que engloba todas las formas de inconstancia climática, cualquiera que sea su naturaleza estadística (o su causa física)" (I.P.C.C., 1995, citado por Cuadrat y Pita, 2000).

Ante esta perspectiva uno pensaría que efectivamente estamos en presencia de un cambio climático, o al menos de uno de sus elementos, pero si se recurre a definiciones acunadas por Hann Julius y citadas por Weber (1951), esta idea cambia radicalmente, ya que lo definen de la siguiente manera "cambio climático se debe entender como una 
modificación permanente en un solo sentido, de larga duración, de modo tal que el clima va empeorando o bien mejorando".

Por otra parte y considerando las confusiones que puede generar el uso erróneo de los términos o las definiciones poca precisas, nos hemos preguntado cual sería el vocablo adecuado para referirnos a los períodos húmedos y secos aquí detectados.

A lo largo del informe se ha empleado términos tales como: período, variación, fluctuaciones, oscilaciones y anomalías.

Si se emplea el término "Período", el mismo debe entenderse según Cuadrat y Pita. (2000) como "un intervalo de tiempo entre máximos y mínimos sucesivos, constantes o casi constantes durante todo la serie de observaciones", situación que, tal como se ha podido apreciar no se desarrolla en nuestro espacio de estudio ni en la serie estadística trabajada puesto que los intervalos positivos y negativos no presentan igual duración.

Por su parte "anomalía" denota una irregularidad o un comportamiento anormal con respecto a lo habitual. Esto genera desconcierto puesto que, tal como lo hemos visto, lo habitual es una sucesión entre estadíos húmedos y secos.

Otros de los vocablos empleados en este trabajo han sido trabajados por Pérez, M. E. (2006), como ser oscilación, variación y fluctuación climática las cuales se consignan a continuación:

-Oscilación climática, aquellas variaciones que abarcan una escala temporal de varias décadas a siglos y milenios,

- Variación climática, cuando las fluctuaciones son lo suficientemente largas como para influir en una media de 30 años, y

- Fluctuación climática, las diferencias de corto período de duración (por ejemplo estacional, interanual, decenal).

Esto denota que el término más adecuado a emplear para referirse no sólo a las variaciones del clima sino también a la sucesión de estadíos húmedos y secos de la precipitación, y que, en definitiva, disminuyen las posibilidades de confusiones, es "fluctuación pluviométrica".

\section{BIBLIOGRAFÍA:}

- BARRY, R. y CHORLEY, R. (1972). "Atmósfera, Tiempo y Clima". Barcelona, Omega.

- BRUNIARD, E. D. (1992). "El ámbito subtropical en la República Argentina (Climatología dinámica y límites climáticos". En: Estudios Geográficos. Madrid, Revista publicada por el Instituto de Economía y Geografía Aplicadas, № 208.

- CONRAD, V. (1941). "The variability of precipitation". En: Monthly Weather Review No 69. Pág. 511.

- CUADRAT, J. y PITA, M. (2000). “Climatología”. 2da Edición. Madrid.

- DEWAR, Robert E. y WALLIS, James R. (1999). "Geographical Patterning of Interannual Rainfall Variability in the Tropics and Near Tropics: An L-Moments Approach". En: Journal of Climate, Volumen 12, № 12. Pág. 3457-3466.

- FERNANDEZ GARCIA, Felipe (1996). "Manual de Climatología Aplicada. Clima, Medio Ambiente y Planificación". Madrid.

- MINETTI, J. y VARGAS, W. (1997). Trends and Jumps in the annual precipitation in South America, south of the $15^{\circ}$ Sur. En: Atmósfera. México, Vol. 11, № 4, pp. 205/221.

Publicado en formato digital: Prof. Claudia Gómez y Prof. María Emilia Pérez. LA VARIABILIDAD PLUVIOMÉTRICA EN LA PROVINCIA DEL CHACO 1955 Y 2009. Revista Geográfica Digital. IGUNNE. Facultad de Humanidades. UNNE. Año 8. № 16. Julio - Diciembre 2011. ISSN 1668-5180 Resistencia, Chaco. En: http://hum.unne.edu.ar/revistas/geoweb/default.htm 
- NICHOLLS, N. (1988). "El Niño Southern Oscilaltion and Rainfall Variability". En: Journal of Climate, Volumen $1, \mathrm{~N}^{\circ} 4$.

- NICHOLLS, N. and K. K. WONG (1990). "Dependence of rainfall variability on mean rainfall, latitude, and the Southern Oscillation". En: Journal of Climate $N^{0}$ 3. Pág. 163-170

- LÓPEZ BERMÚdEZ, F; RUBIO RECIO, J; CUADRAT J. (1992). "Geografía Física". Cátedra. Madrid. Pág. 594

- PÉREZ M. E. (2006). "Fluctuaciones Climáticas Y Variabilidad Temporal Del Clima En El Norte Argentino - 1931/2005". Revista Geográfica Digital. IGUNNE. Facultad de Humanidades. UNNE. Año 3. № 6. Julio - Diciembre.

- ROPELEWSKI, CH. and HALPERT, S. (1987). "Global and regional precipitation patterns associated with the El Niño/Southern Oscillation". En: Monthly Weather Review. № 115. Pág. 1606-1626.

\section{PÁGINA WEB:}

-A.P.A (Administración Provincial del Agua). (www.chaco.gov.ar/apa ). 